\title{
High-Speed Concatenation of Frequency Ramps Using Sampled Grating Distributed Bragg Reflector Laser Diode Sources for OCT Resolution Enhancement
}

\author{
Brandon George and Dennis Derickson \\ California Polytechnic State University, Electrical Engineering Department \\ 1 Grand Avenue, San Luis Obispo,CA 93407 USA ddericks@calpoly.edu
}

\begin{abstract}
Wavelength tunable sampled grating distributed Bragg reflector (SG-DBR) lasers used for telecommunications applications have previously demonstrated the ability for linear frequency ramps covering the entire tuning range of the laser at $100 \mathrm{kHz}$ repetition rates ${ }^{1}$. An individual SG-DBR laser has a typical tuning range of $50 \mathrm{~nm}$. The InGaAs/InP material system often used with SG-DBR lasers allows for design variations that cover the 1250 to $1650 \mathrm{~nm}$ wavelength range. This paper addresses the possibility of concatenating the outputs of tunable SGDBR lasers covering adjacent wavelength ranges for enhancing the resolution of OCT measurements. This laser concatenation method is demonstrated by combining the $1525 \mathrm{~nm}$ to $1575 \mathrm{~nm}$ wavelength range of a "C Band" SG-DBR laser with the 1570nm to $1620 \mathrm{~nm}$ wavelength coverage of an "L-Band" SG-DBR laser. Measurements show that SGDBR lasers can be concatenated with a transition switching time of less than $50 \mathrm{~ns}$ with undesired leakage signals attenuated by $50 \mathrm{~dB}$.

Keywords: Distributed Bragg reflector diode lasers, optical coherence tomography.
\end{abstract}

\section{INTRODUCTION}

The single-chip SG-DBR wavelength-tunable laser has been shown ${ }^{1}$ to be capable of a $100 \mathrm{kHz}$ repetition rate continuous wavelength ramp or a $10 \mu$ s single-shot sweep time. The wavelength ramp consists of fifty 1 -nm wide continuous tuning segments that are combined to produce the full 1525 to $1575 \mathrm{~nm}$ range. The spectral output of these tunable sources provides single longitudinal mode outputs with a typical spectral linewidth of $100 \mathrm{MHz}$. The existing high volume production of the SG-DBR laser in the telecommunication industry and small source size gives the opportunity for cost-effective and portable OCT applications. In many OCT applications, it is beneficial to have a wider source tuning range for enhanced distance resolution in OCT. The InGaAs/InP semiconductor material system used by telecommunications SG-DBR lasers offers design variations that can cover any wavelength in the 1250 to $1650 \mathrm{~nm}$ range. This paper shows that adjacent wavelength coverage SGDBR tunable lasers can be easily concatenated due to the unique architecture of the laser. The Semiconductor Optical Amplifier (SOA) that is included as the output segment of this single chip laser design can be used as a fast optical switch to aid the concatenation of adjacent wavelength tuning segments. It will be shown that the concatenation of adjacent wavelength sweep segments can be accomplished with a dead-time of less than $50 \mathrm{~ns}$ using the SOA that is monolithically integrated on the laser chip. In order to demonstrate concatenation of SGDBR lasers into an extended frequency ramp versus time a "C-Band" SGDBR laser and an "LBand" SGDBR laser were characterized. Each single chip SGDBR laser has 5 control segments as illustrated in figure 1.

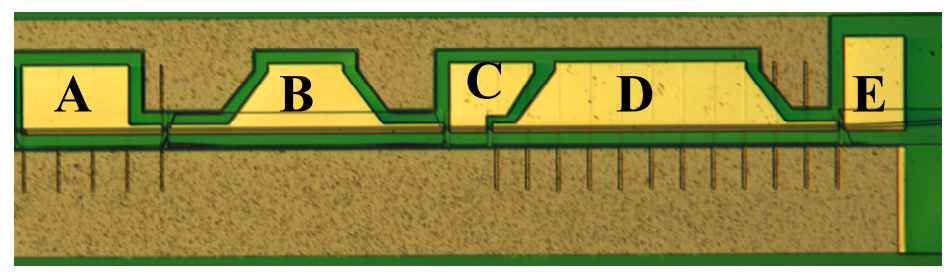

Figure 1. This is a top view of a single-chip Sampled Grating Distributed Bragg Reflector Laser (SGDBR) used for OCT measurements. Currents in Back Mirror (A), Front Mirror (D), and Phase Section (C), control the laser frequency. Currents in the gain section (B) and the semiconductor optical amplifier (E) control the laser power. The chip dimensions are $0.5 \mathrm{~mm}$ by $3 \mathrm{~mm}$.

Optical Coherence Tomography and Coherence Domain Optical Methods in Biomedicine XIV, edited by Joseph A. Izatt, James G. Fujimoto, Valery V. Tuchin, Proc. of SPIE Vol. 7554, 75542O - ( 2010 SPIE CCC code: $1605-7422 / 10 / \$ 18 \cdot$ doi: $10.1117 / 12.842604$ 
In order to construct a frequency ramp using SGDBR lasers, the laser wavelength as a function of drive current to the front mirror, back mirror and phase sections needs to be obtained. Figure 2 shows the results of wavelength mapping as a function of drive current to the front and back mirror segments of an L-Band SGDBR laser. Figure 3 shows the results of wavelength mapping as a function of drive current to the front and back mirror segments of a C-Band SGDBR laser.

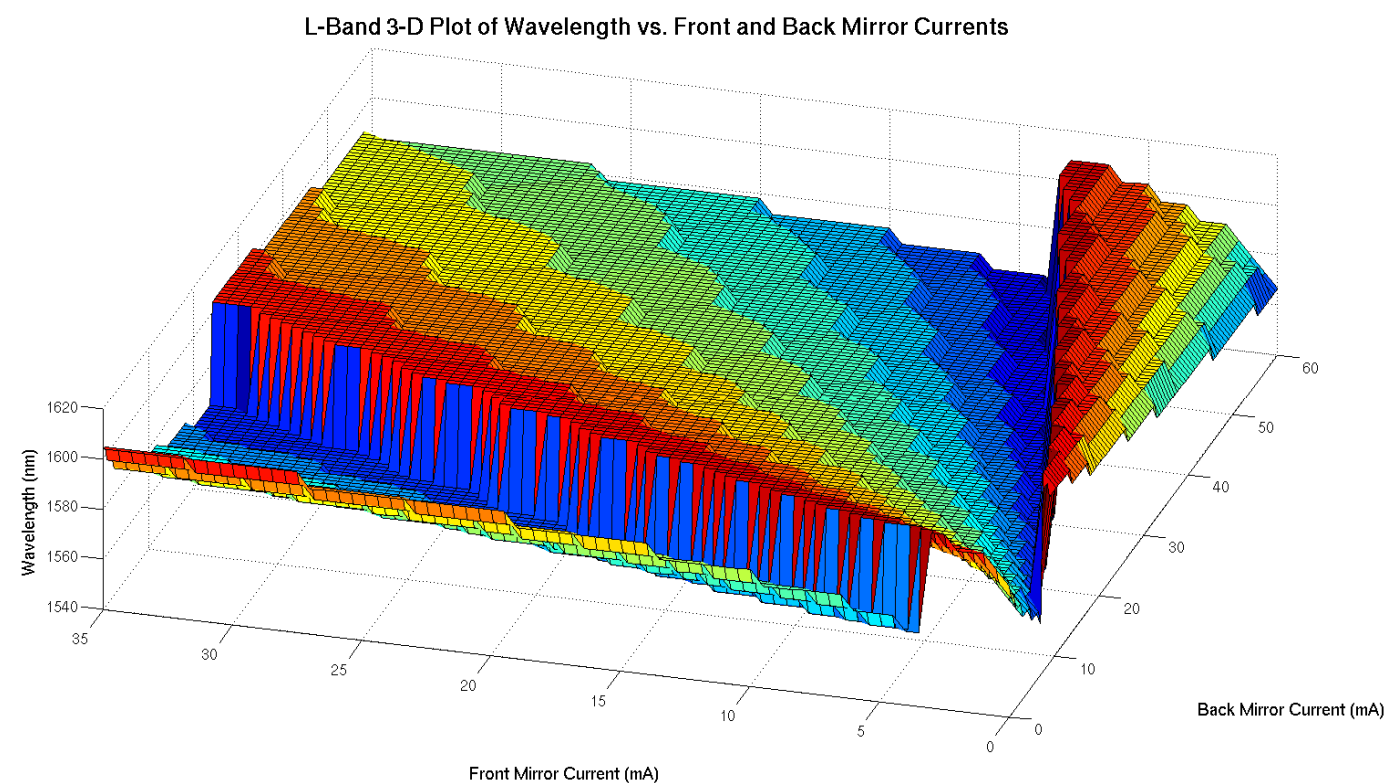

Figure 2. Three-dimensional (mode map) plot of wavelength versus front mirror and back mirror currents for the L-band laser. The approximate wavelength coverage is from $1565 \mathrm{~nm}$ to $1620 \mathrm{~nm}$.

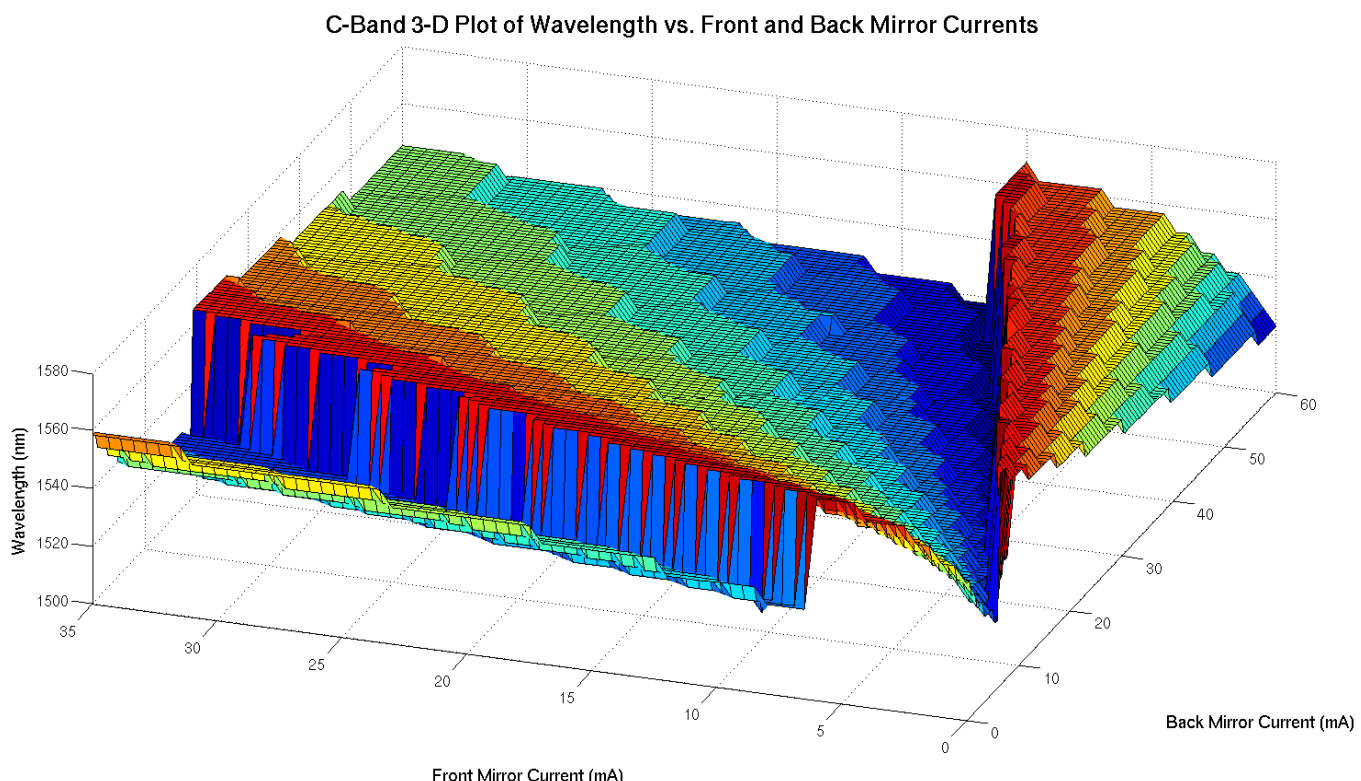

Figure 3. Three-dimensional (mode map) plot of wavelength versus front mirror and back mirror currents for the L-band laser. The approximate wavelength coverage is from $1525 \mathrm{~nm}$ to $1570 \mathrm{~nm}$.

In order to create a linear frequency ramp versus time, one needs to map a sequence for front and back mirror current values that allow the laser to tune over its entire frequency range in a linear ramp versus time. Figure 4 shows the 
current paths that were chosen in order to tune over the range of the L-Band laser. Figure 5 shows the current paths that were chosen in order to tune over the range of the C-Band laser. In the construction of a linear frequency versus time ramp from the concatenated $\mathrm{C}$ and $\mathrm{L}$ band lasers it was decided to sweep from lowest frequency to highest frequency. This means that paths 1 through 8 are first transited in figure 4. Paths 1-8 of figure 5 are then concatenated to form the entire frequency ramp.

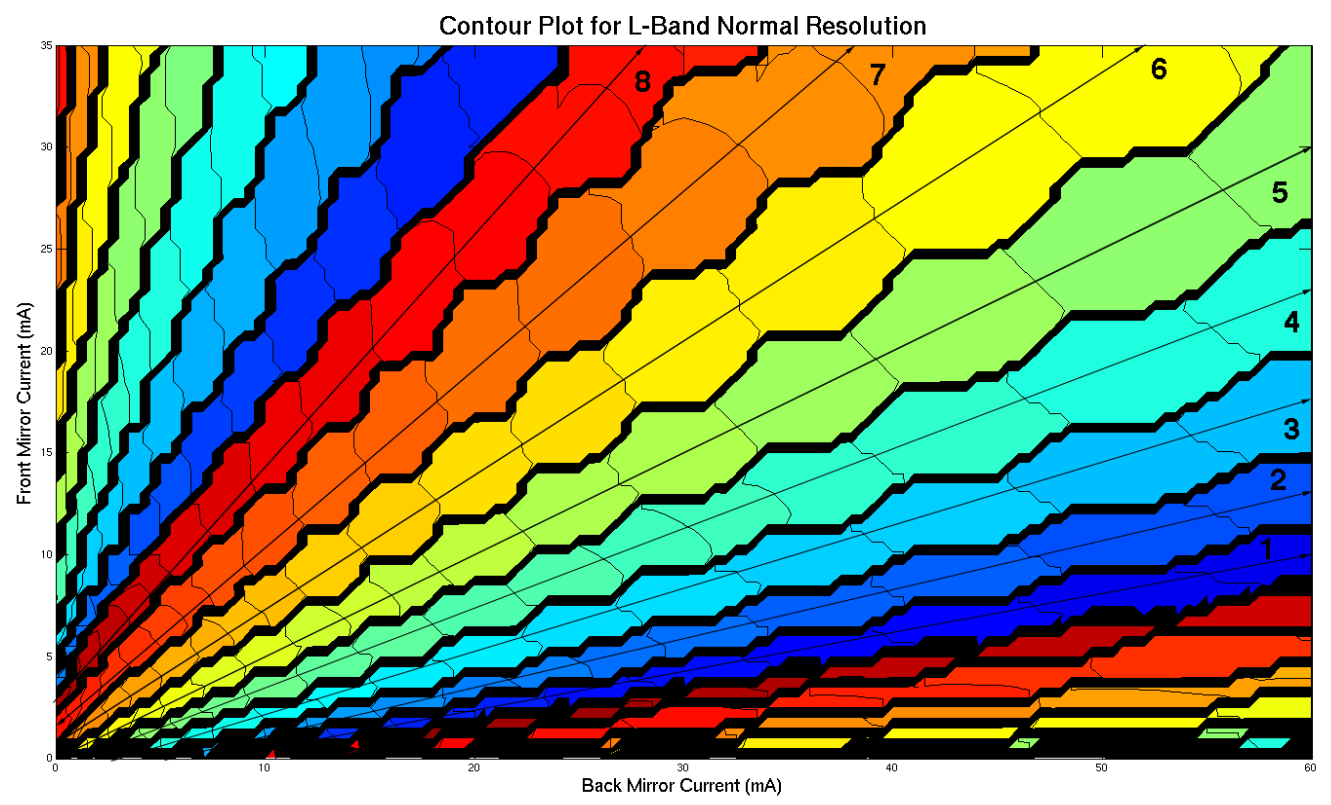

Figure 4. This plot contains similar information to that of figure 2 for the L-Band laser from a top view. Lines 1 through 8 illustrate the path of the current versus time in order to accomplish a linear frequency ramp versus time.

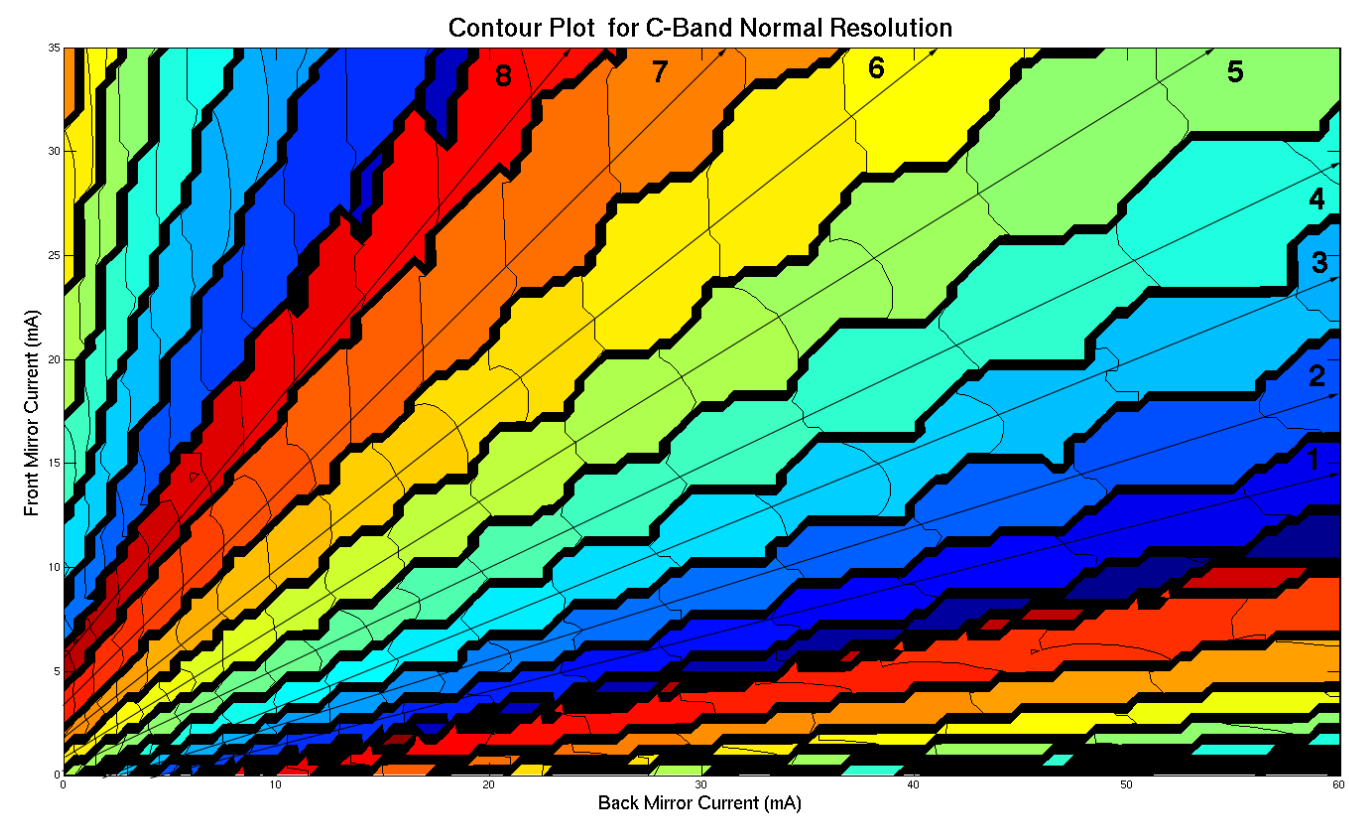

Figure 5. This plot contains similar information to that of figure 3 for the C-Band laser from a top view. Lines 1 through 8 illustrate the path of the current versus time in order to accomplish a linear frequency ramp versus time. 


\section{DESCRIPTION OF THE CONCATENATION PROCESS}

Figure 6 shows the system block diagram used to generate separate wavelength sweeps from two SGDBR lasers and the method for sweep concatenation.

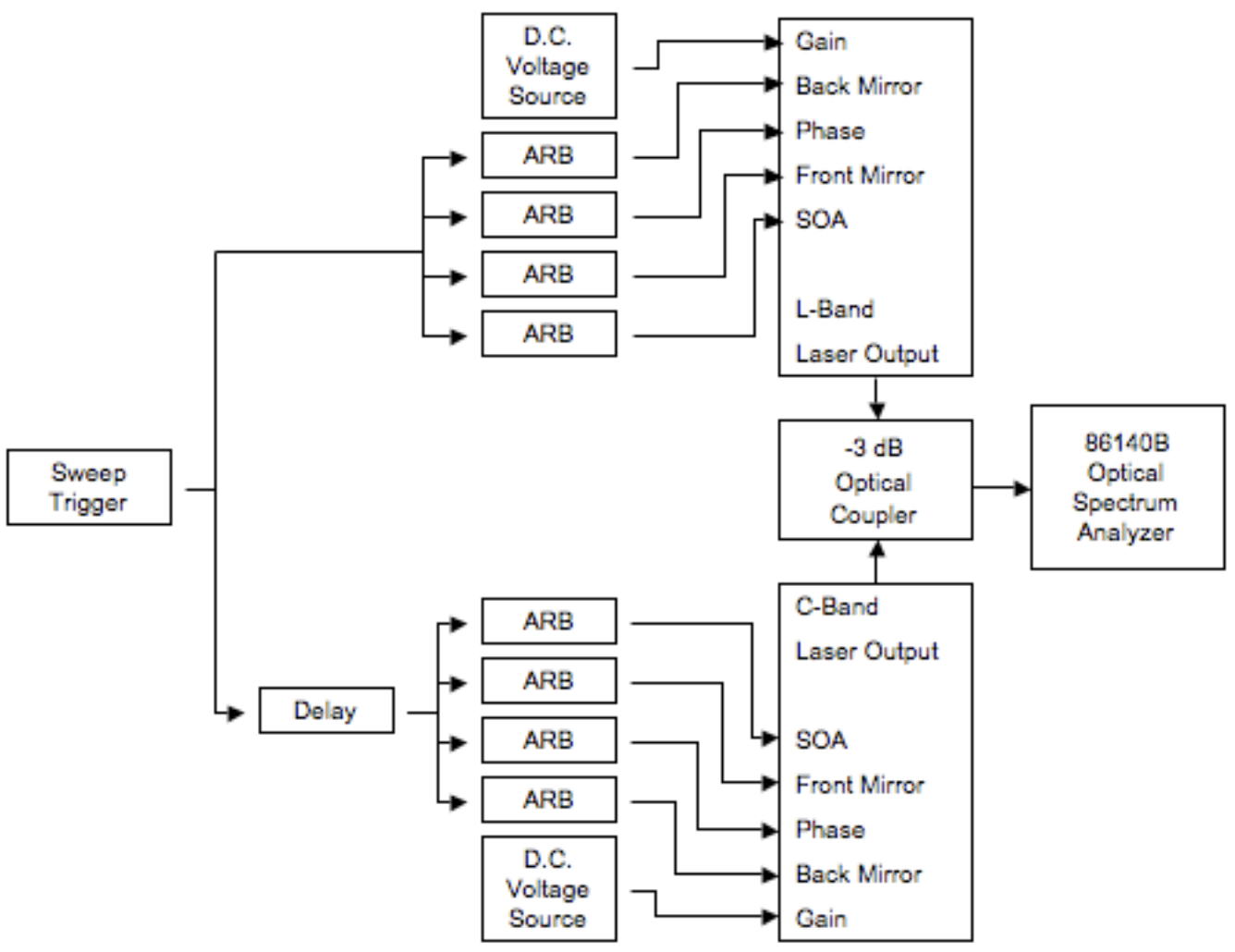

Figure 6. This is the block diagram for generating a wide spectral-width linear frequency versus time ramp by concatenating two SG-DBR lasers.

The top laser block is an "L-Band" SGDBR laser that covers the nominal wavelength range of 1565 to $1620 \mathrm{~nm}$. The bottom laser block is a "C-Band" SGDBR laser that covers the nominal wavelength range of 1525 to $1575 \mathrm{~nm}$. Each monolithic SG-DBR laser chip (see figure 1) has three segments that control the laser wavelength; the Back Mirror $(\mathrm{BM})$, Front Mirror (FM), and Phase $(\phi)$. Each of the laser segments is driven by an Arbitrary Waveform Generator (ARB) to control the current versus time into each segment of the laser. The ARBs have a clock frequency of $50 \mathrm{MHz}$ with an effective $20 \mathrm{MHz}$ of analog bandwidth. The gain segment is DC biased and provides overall gain for the laser. The Semiconductor Optical Amplifier (SOA) boosts the output power from the laser. The SOA will also be used as a fast optical switch for concatenation of the outputs of the L-Band and C-Band SG-DBR devices. The SOA can also be used to level the power versus time at the output of the laser during the sweep. Each of the ARBs are time synchronized by a master sweep trigger signal. A programmable delay is used to control the switching point in time between the LBand and C-Band laser operation in order to concatenate the sweep of the two lasers. Finally the outputs of the two lasers are combined in a wavelength independent coupler with the output analyzed by measurement instrumentation. The wavelength coverage of the laser is first monitored in the optical spectrum analyzer. The linearity of the frequency versus time from the laser can be monitored by replacing the optical spectrum analyzer with a repetitive pass band optical filter, a photodetector and an oscilloscope. 
Figure 7 illustrates the current versus time that is provided for each segment of the two SGDBR sources. The wavelength tuning map for each SGDBR laser must first be mapped as a function of current in the FM, BM and $\phi$ segments $^{2}$. In order to provide a linear ramp in frequency from the laser, the saw-tooth waveforms labeled L-FM, L$\mathrm{BM}$, and L- $\phi$ must be synthesized from the previously generated wavelength-tuning maps shown in figures 2-5. Each vertical abrupt change in the segment current versus time represents an abrupt change in current that causes the laser to jump between different longitudinal modes but with no associated wavelength shift. The individual output from each SG-DBR laser has approximately 50 continuous tuned segments that are joined together for the full wavelength sweep.

Figure 7 also shows the C-FM, C-BM and C- $\phi$ waveforms for the C-Band wavelength sweep. In this case the output of the C-Band laser is delayed in time so that the sweep starts after the finish of the L-Band sweep.

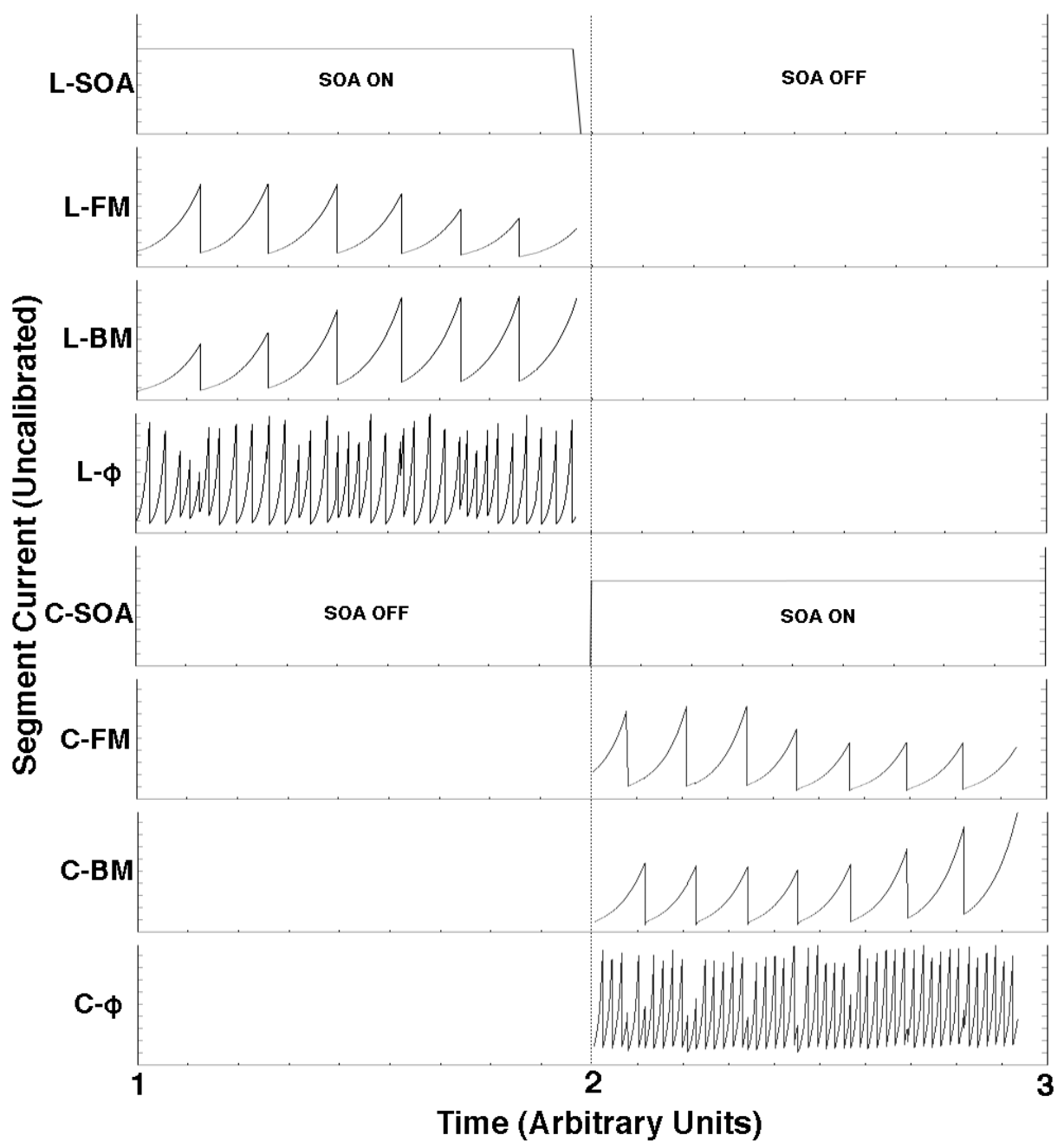

Figure 7. The current versus time into the SG-DBR laser segments for a concatenated C-Band and L-Band wavelength sweep is shown.

At time $=1$ the master sweep trigger initiates the sweep. The L-SOA signal is on allowing a high output power from the L-Band Laser. The C-SOA is off resulting in a large attenuation of the C-Band laser output. The sawtooth current waveforms found in L-FM, $\mathrm{L}-\mathrm{BM}$, and L- $\phi$ segments then create a frequency ramp in the L-Band laser output.

At time $=2$, the L-SOA is tuned off and the C-SOA is turned on. The sawtooth C-FM, C-BM and C- $\phi$ current waveforms then create a frequency ramp in the C-Band laser. Point 1 represents the trigger signal for the L-Band laser ARBs, while Point 2 represents the trigger signal for the C-band laser ARBs. For single shot operation, the sweep will end at time=3. For repetitive sweeps, the operation will reset back to time $=1$. Sweep-back time of the laser is very fast and is presently limited by the $50 \mathrm{~ns}$ rise time of the ARB drive waveform. 
The C-Band and L-Band wavelength sweeps associated with the current waveforms shown in figure 7 are shown in Figure 8.

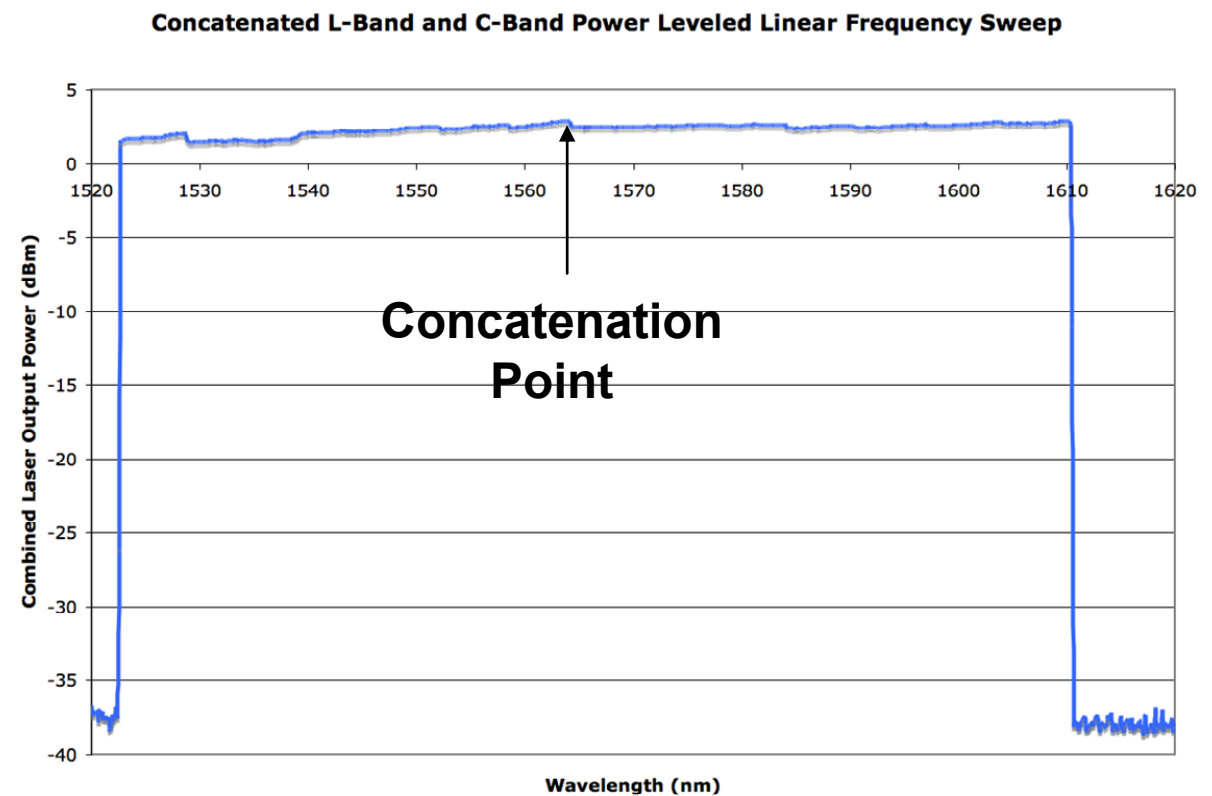

Figure 8. The output power versus wavelength for both the C-Band and L-Band SG-DBR lasers for the current drive waveforms shown in figure 7.

The L-Band wavelength coverage was from $1560.702 \mathrm{~nm}$ to $1610.092 \mathrm{~nm}$ or $49.4 \mathrm{~nm}$ of bandwidth, The C-band laser tuning shape consisted of eight wavelength-tuning paths from $1523.040 \mathrm{~nm}$ to $1569.970 \mathrm{~nm} \mathrm{or} 46.9 \mathrm{~nm}$ of bandwidth. However, since path 8 of the C-band laser completely overlaps with the L-band laser sweep, it was omitted from the final tuning dictionary. What remained was a constant C-band wavelength sweep from $1523.064 \mathrm{~nm}$ to $1564.166 \mathrm{~nm}$ or $41.1 \mathrm{~nm}$ of bandwidth. A wavelength concatenation point of $1564 \mathrm{~nm}$ was used for this experiment.

\section{CHARACTERIZING THE L-BAND/C-BAND CONCATENATION POINT}

Each SG-DBR laser is configured to construct linear wavelength or frequency sweeps with approximately fifty $1 \mathrm{~nm}$ wide modal segments that are stitched together. By concatenating two wavelength sweeps together, we are introducing a new concatenation stitching point between the two lasers. The major difference between the modal stitching points in the laser and the concatenation stitching point is the control segment of the laser. Modal switching points use the FM, $\mathrm{BM}$ and $\phi$ segments of the laser. Concatenation stitching points use the SOA segments of the laser. When the SOA segment current is on, high output power is delivered. When the SOA segment current is off, the laser output is attenuated. Figure 9 illustrates the concatenation stitching process. For the first part of the sweep the L-SOA is on and the C-SOA is off. In the second part of the sweep the C-SOA is on and the L-SOA is off. The switching characteristics of the SOA are key to the successful concatenation of wavelength sweeps. The next two figures will explore the switching speed and the attenuation levels that are possible with the SOA switch that is part of the monolithic structure of the SG-DBR laser.

Figure 9 shows a measurement of the optical power versus time that is measured both at the L-Band Output, the CBand output and the combined output from the laser. In this case a high bandwidth photodetector and an oscilloscope replace the OSA measurement block found in Figure 6. 


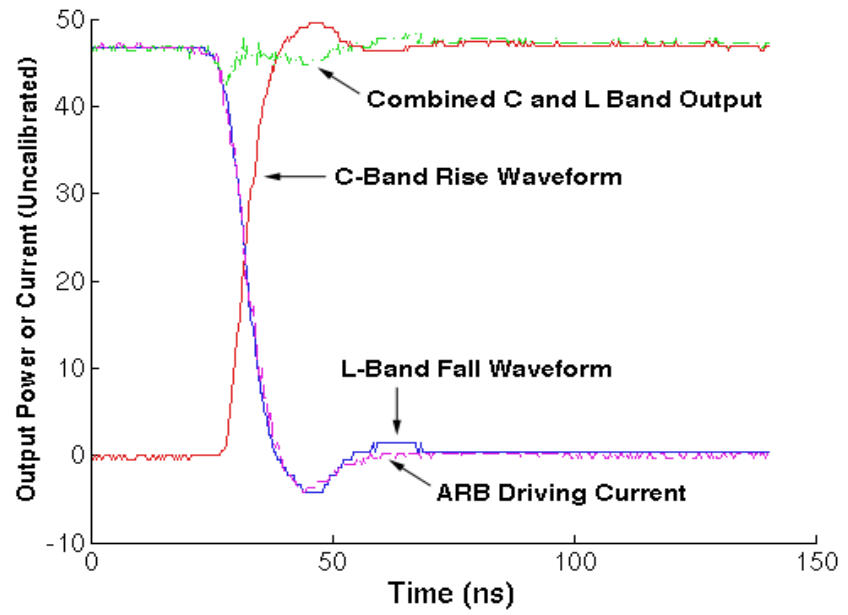

Figure 9. The output power versus time is shown for various outputs in the block diagram of figure 6. The C-Band, L-Band and combined optical output power versus time are shown. In addition the electrical drive current (ARB Driving Current) waveform to the SOA segment is also shown.

The L-Band optical output power goes from being on to off in a period of less than $50 \mathrm{~ns}$. The C-Band output power goes from being off to on in a period of less than $50 \mathrm{~ns}$. The measurement of the drive waveform to the SOA segment (labeled ARB driving current) illustrates that the rise and fall times are primarily driven by the rise and fall time drive capabilities of the ARB circuitry in this example. Figure 9 also shows the combined optical power from the wavelength independent combiner. A summary from this measurement is that very fast switching times can be accomplished between L-Band and C-Band operations using the SOA segment. The SOA segment is part of the monolithic laser structure so no extra part count (and associated cost) is needed for this switching other than the wavelength independent optical coupler and an additional ARB for each laser.

The SOA switch is not ideal though and there is remnant signal leakage from an SG-DBR laser when the SOA is in its off state. Figure 10 shows a measurement of the C-Band laser output power versus wavelength for the SOA in its on state and in its off state.

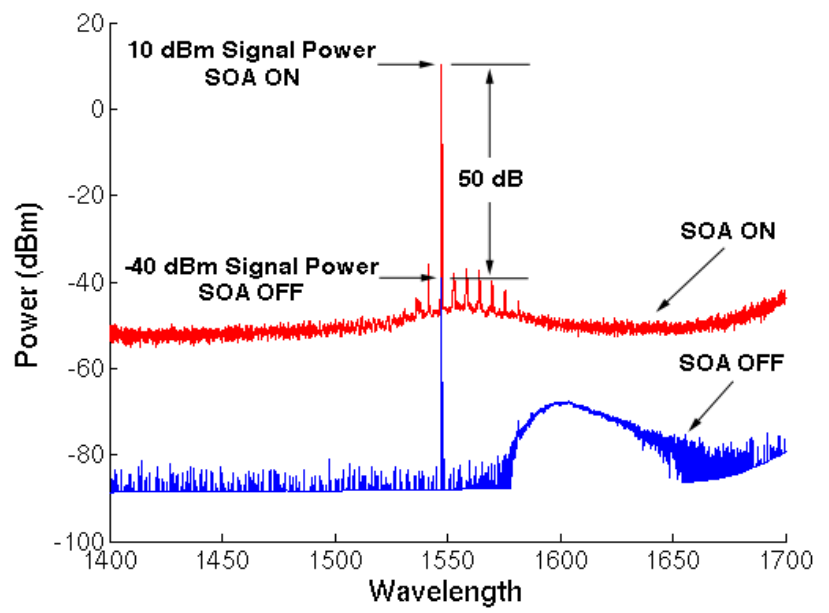

Figure 10. The output of the C-Band laser is shown as a function of wavelength both in the on state and the off state. The resolution bandwidth of the OSA is $0.07 \mathrm{~nm}$ in this measurement. 
When the SOA segment is in its "on state", it delivers $10 \mathrm{dBm}(10 \mathrm{~mW})$ of signal power. The integrated "on state" amplified spontaneous emission (ASE) power of the laser from 1525 to $1575 \mathrm{~nm}$ is estimated as $-13.5 \mathrm{dBm}(45 \mu \mathrm{W})$. The "off state" signal power is $-40 \mathrm{dBm}(0.1 \mu \mathrm{W})$. The "off state" ASE power is estimated as $-41.8 \mathrm{dBm}(0.067 \mu \mathrm{W})$. The "off state" ASE power at wavelengths longer than $1570 \mathrm{~nm}$ has reduced attenuation because the photon energy at these wavelengths is less than the band gap energy of the material in the SOA segment.

The critical point to be taken from the data of figure 5 is the amount of undesired power that leaks into the output with the SOA off compared to the desired signal. In this case the "on state" would have a signal power of $10 \mathrm{dBm}$. The undesired leakage signal would be dominated by the $-40 \mathrm{dBm}$ source signal from the attenuated laser. So the undesired lasing signal will be $50 \mathrm{~dB}$ lower that of the desired laser output. The attenuation of the SOA is sufficient so that it will have a negligible effect on the combined output shown in figure. 1.

\section{SUMMARY}

The concatenation of two wavelength tunable SG-DBR wavelength sweeps was illustrated. The SOA segment of the SG-DBR laser is key to cost-effective frequency sweep concatenation. The switching time for sweep concatenation was found to be less than $50 \mathrm{~ns}$, limited by the ARB rise time capability. The attenuation of the SOA segment is sufficient so that only a $50 \mathrm{~dB}$ attenuated signal leaks into the combined output. The result shows that SG-DBR lasers can successfully concatenated offering better time resolution for OCT measurements. It should be possible to concatenate a large number of adjacent SG-DBR frequency sweeps to cover the $1250 \mathrm{~nm}$ to $1650 \mathrm{~nm}$ wavelength range that is possible with the SG-DBR laser using the InGaAsP/InP material system. The high ramp repetition rate, low cost and small size of the concatenated source makes this an attractive option for low cost and portable OCT applications with improved distance resolution.

\section{REFERENCES}

[1] Shane O'Connor, Michael A. Bernacil, Andrew DeKelaita, Ben Maher, and Dennis Derickson, “100 kHz Axial Scan Rate Swept-Wavelength OCT using Sampled Grating Distributed Bragg Reflector Lasers," in Coherence Domain Optical Methods and Optical Coherence Tomography in Biomedicine XIII, edited by Joseph A. Izatt, James G. Fujimoto, Valery V. Tuchin, Proceedings of SPIE Vol. 7168 ( 2009).

[2] Shane O'Connor, Michael A. Bernacil, and Dennis Derickson, "Generation of High Speed, Linear Wavelength Sweeps Using Sampled Grating Distributed Bragg Reflector Lasers,” IEEE LEOS Annual Meeting, Newport Beach, CA, paper TuB2 (2008). 